\title{
Pretreatment DWI with Histogram Analysis of the ADC in Predicting the Outcome of Advanced Oropharyngeal Cancer with Known Human Papillomavirus Status Treated with Chemoradiation
}

\author{
(D) M. Ravanelli, (D) A. Grammatica, (D) M. Maddalo, (D) M. Ramanzin, (D) G.M. Agazzi, (DE. Tononcelli, (D) S. Battocchio, (D). Bossi, \\ (D) M. Vezzoli, (D) R. Maroldi, and (DD. Farina
}

\begin{abstract}
BACKGROUND AND PURPOSE: The incidence of oropharyngeal squamous cell carcinoma (OPSCC) has increased in the period from the 1970s to 2004, due to increase of infection with human papilloma virus (HPV). This study aimed to examine the role of histogram analysis of the ADC in treatment response and survival prediction of patients with oropharyngeal squamous cell carcinoma and known human papillomavirus status.
\end{abstract}

MATERIALS AND METHODS: This was a retrospective single-center study. Following inclusion and exclusion criteria, data for 59 patients affected by T2-T4 (according to the 8th edition of the AJCC Cancer Staging Manual) oropharyngeal squamous cell carcinoma were retrieved. Twenty-eight had human papillomavirus-positive oropharyngeal squamous cell carcinoma, while 31 had human papillomavirus-negative oropharyngeal squamous cell carcinoma. All patients underwent a pretreatment MR imaging. Histogram analysis of ADC maps obtained by DWI $\left(b=0-1000 \mathrm{~mm} / \mathrm{s}^{2}\right)$ was performed on the central section of all of tumors. The minimum followup period was 2 years. Histogram ADC parameters were associated with progression-free survival and overall survival. Univariable and multivariable Cox models were applied to the data; $P$ values were corrected using the Benjamini-Hochberg method.

RESULTS: At univariable analysis, both human papillomavirus status and mean ADC were associated with progression-free survival (hazard ratio $=0.267, P<.05$, and hazard ratio $=1.0028, P \leq .05$, respectively), while only human papillomavirus status was associated with overall survival (hazard ratio $=0.213, P \leq .05$ ) before correction. At multivariable analysis, no parameter was included (in fact, human papillomavirus status lost significance after correction). If we separated the patients into 2 subgroups according to human papillomavirus status, ADC entropy was associated with overall survival in the human papillomavirus-negative group (hazard ratio $=4.846, P=.01)$.

CONCLUSIONS: ADC and human papillomavirus status are related to progression-free survival in patients treated with chemoradiation for advanced oropharyngeal squamous cell carcinoma; however, this association seems to result from the strong association between ADC and human papillomavirus status.

ABBREVIATIONS: HPV = human papillomavirus; OPSCC = oropharyngeal squamous cell carcinoma; OS = overall survival; PFS = progression-free survival; TNM = Tumor, Node, Metastasis

I:

the United States, the incidence of oropharyngeal squamous cell carcinoma (OPSCC) has increased from the 1970s to $2004,{ }^{1}$ despite successful effort to control alcohol and tobacco abuse. The increase is due to the increasing incidence of OPSCC related to infection with Human Papillomavirus (HPV), in particular,

Received February 4, 2020; accepted after revision May 23

From the Departments of Radiology (M. Ravanelli, M. Ramanzin, G.M.A., E.T., R.M., D.F.), Otorhinolaryngology (A.G.), Radiation Oncology (M.M.), Pathological

Anatomy (S.B.), and Medical Oncology (P.B.), and Molecular and Translational Medicine (M.V.), University of Brescia, Brescia, Italy.

Please address correspondence to Marco Ramanzin, MD, Department of Radiology, University of Brescia, Piazzale Spedali Civili 1, 25123, Brescia, Italy; e-mail: marco.ramanzin91@gmail.com

http://dx.doi.org/10.3174/ajnr.A6695 among younger men who did not smoke or consume alcohol excessively.

Along with the increasing incidence of OPSCC, survival rates have improved. In fact, HPV-related OPSCC is a separate entity compared with HPV-negative OPSCC, with different biologic behavior and better outcome. Thus, HPV-positive and HPV-negative OPSCC have 2 distinct Tumor, Node, Metastasis (TNM) systems in the 8th edition of the AJCC Cancer Staging Manual, ${ }^{2-4}$ and dose de-intensification protocols for HPV-positive OPSCC are being investigated in clinical trials. ${ }^{5}$

HPV status has, thus, become one of the most important factors in predicting survival in patients with OPSCC. However, several methods are being investigated to better stratify the risk of 
treatment failure. ${ }^{6,7}$ The predictive nomograms obtained from these studies, including clinical, social, and educational variables, have proved valid in the geographic context in which they were developed but are not replicable in populations with different geographic provenances. ${ }^{8}$ Thus, more objective biomarkers or surrogates are required to predict response to treatment and survival and to build more reproducible models.

Radiomics is a "big data" approach based on the extraction of several quantitative features from diagnostic images (mainly CT images), which are then used to build predictive and prognostic models. Leijenaar et $\mathrm{al}^{9}$ developed and externally validated a CT radiomics signature for predicting survival in patients with OPSCC without taking into account other clinicopathologic data, HPV included. The M.D. Anderson Cancer Center Head and Neck Quantitative Imaging Working Group developed a CT-based radiomics signature, which, together with HPV status, age, sex, and smoking status, predicted survival (unfortunately, HPV status was not known in more than half of the patients). ${ }^{10}$

Radiomics is currently a complex and computationally expensive approach, and further studies are needed to translate these into clinical scenarios. Furthermore, all of these studies are based on CT images, while in some centers, MR imaging is used to more accurately stage OPSCC. At the present time, no radiomics studies based on MR imaging have been performed in this setting.

DWI is an MR imaging sequence that explores tissue microarchitecture, relying on water motion within it, which is quantitatively described by the ADC. This sequence is currently part of most of the standard protocols in head and neck cancer studies.

As will be discussed later in more detail, ADC has been used as a potential predictor of outcome in head and neck cancer. ${ }^{11}$ However, in these studies, relevant tumor characteristics have usually been omitted, hampering the clinical utility of their results. ADC maps can be easily segmented and expressed as a mean value or, in more detail, described by first-order statistics (histogram analysis).

This study aimed to ascertain the role of ADC histogram analysis in the treatment response and survival prediction of patients with OPSCC with known HPV status.

The hypothesis that gives origin to this study is that the role of ADC in patient stratification is overestimated when HPV status is not known; the latter (whose predictive role is well-established) is strongly associated with the former.

\section{MATERIALS AND METHODS \\ Patients}

This was a single-center retrospective study including patients with histologically proved T2-T4 OPSCC who underwent chemoradiation with curative intent after an individual assessment performed by our internal multidisciplinary team. Patients were enrolled between March 2010 and April 2017 to guarantee a minimum 2-year follow-up period. Inclusion criteria were the following: patients with pretreatment head and neck MR imaging with sufficient image quality and in whom echo-planar DWI sequences were acquired; tumors with known HPV status; and smoking status. We excluded patients with low-quality MR images because of motion artifacts (nondiagnostic, as assessed by an expert head and neck radiologist); tumors too small to be segmented $(<2 \mathrm{~cm})$; and patients with $<2$ years of follow-up in whom recurrence or death had not occurred. Staging was calculated for each patient according to the 8th edition of AJCC Cancer Staging Manual, Head and Neck Section.

\section{HPV Determination}

HPV status was determined with the digene Hybrid Capture 2 High-Risk HPV DNA test (Qiagen). This is an in vitro nonradioactive nucleic acid hybridization assay with signal amplification using a chemiluminescent microtiter plate. This test is able to detect $18 \mathrm{HPV}$ types, including high-risk $(16,18,31,33,35,39$, $45,51,52,56,58,59,68)$ and low-risk types $(6,11,42,43,44)$. The Hybrid Capture 2 test (Qiagen) shows a sensitivity and specificity equivalent to that of the polymerase chain reaction. DNA analysis was performed independent from p16/INK4a status, which was also assessed.

\section{Chemoradiation Therapy}

Patients were treated with definitive radiochemotherapy, delivered with a linear accelerator using an intensity-modulated technique (intensity-modulated radiotherapy, volumetric modulated arc therapy, or TomoTherapy [SSMHealth]). In the case of conventional fractionation with sequential boost, a dose of $70 \mathrm{~Gy}$ was prescribed to the tumor and the lymph nodes involved, while a dose of $50 \mathrm{~Gy}$ was prescribed to the remaining uninvolved neck levels. In case of treatment planned with a simultaneous integrated boost, slight variations in the number of fractions, total dose, and dose per fraction were administered, with doses equivalent to normo-fractionation. Chemotherapy with concurrent cisplatin was administered weekly at a dose of $40 \mathrm{mg} / \mathrm{m}^{2}$.

\section{MR Imaging Protocol}

MR imaging studies were performed on a $1.5 \mathrm{~T}$ scanner (Magnetom Aera; Siemens) with a dedicated head and neck 20channel phased array coil. The acquisition protocol included the following: T2-weighted TSE sequences on axial and coronal planes (for palatine tonsil cancer) or the sagittal plane (for base of the tongue and posterior wall cancers); a T1-weighted TSE sequence on the axial plane; a 3D fat-saturated gradient recalled-echo sequence (volumetric interpolated brain examination) with isotropic spatial resolution of $0.7 \mathrm{~mm}$ after gadolinium-based contrast agent injection; and DWI with the following parameters: TR $=3900 \mathrm{~ms}$, TE $5=9 \mathrm{~ms}$, section thickness $=$ $3 \mathrm{~mm}$, matrix $=132 \times 132$, b-values $=0$ and $1000 \mathrm{~mm} / \mathrm{s}^{2}$. The ADC was generated automatically. Treatment response was assessed with a second MR imaging study performed 12 weeks after treatment (Fig 1).

\section{Image Analysis}

T2-weighted TSE sequences and DWI sequences with ADC maps were transferred to an off-line PC and analyzed using open-source software (ImageJ; National Institutes of Health). The segmentation was performed by a head and neck radiologist with $>10$ years' experience. An ROI encompassing the whole primary tumor on its largest cross-sectional area was 


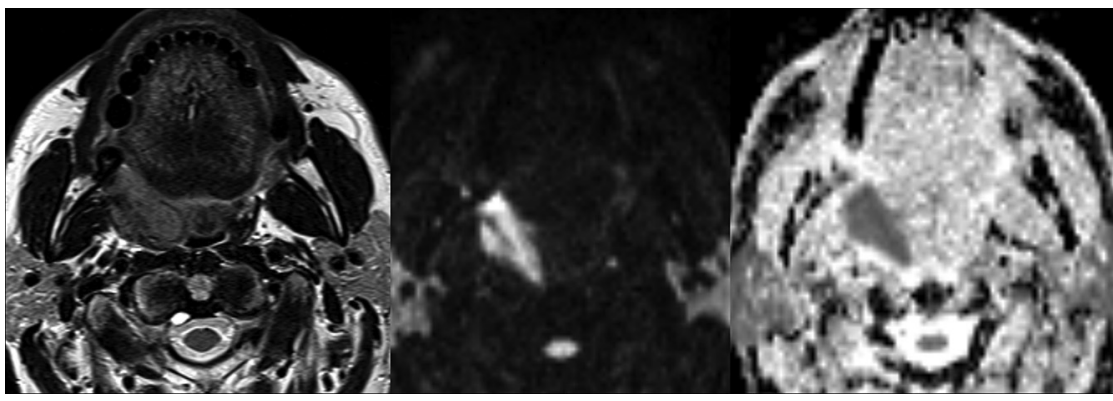

FIG 1. Axial T2, $b=1000$, and ADC map of an OPSCC of the right palatine tonsil.

Table 1: Baseline patient and tumor characteristics

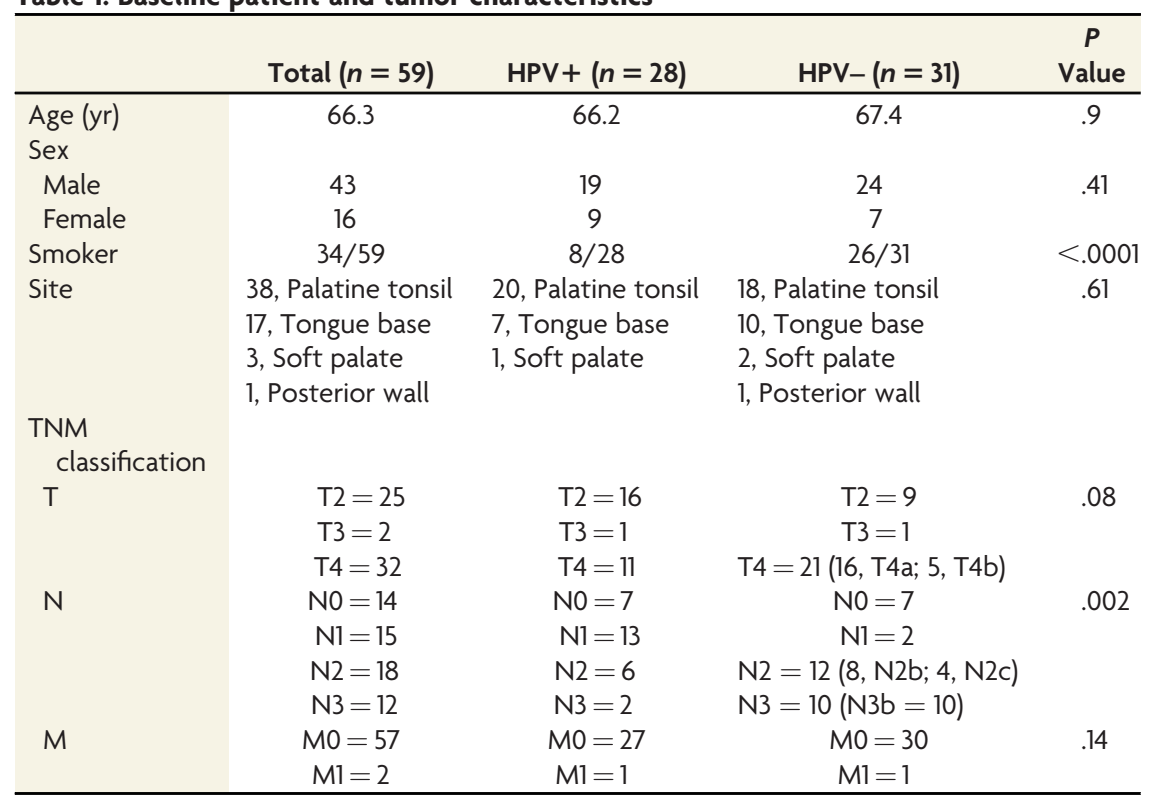

Note:-HPV+ indicates human papillomavirus-positive; HPV-, human papillomavirus-negative.

logistic regression between the histogram parameter of ADC and HPV status was conducted to assess whether any association was present between ADC parameters and HPV. All $P$ values were corrected to minimize the false discovery rate (BenjaminiHochberg correction).

Statistical significance was fixed at $P<.05$. Statistical analysis was performed using MedCalc 16.4.3 (MedCalc Software).

\section{RESULTS}

\section{Patient and Tumor Characteristics}

In total, 68 patients with T2-T4 OPSCC were included according to the inclusion criteria. Of those, 4 patients were excluded because the primary tumor was too small to be segmented; a further 5 patients were excluded because of low image quality due to motion artifacts, as established by an expert head and neck radiologist. Analysis was thus performed on 59 patients (43 men, 16 women). Baseline patient and tumor characteristics are shown in Table 1. Notably, 28 patients had HPV-positive tumors, while 31 had an HPV-negative lesion. Eight of the $28 \mathrm{HPV}$-positive patients were smokers, while the incidence rose to 26/31 patients with HPV-negative tumors $(P>.0001)$.

drawn on ADC maps; the segmentation was aided using sideby-side visualization of T2-weighted and $b=1000$ images. We calculated the following histogram parameters on ADC maps: mean, SD, kurtosis, skewness, and entropy. "Kurtosis" indicates the histogram peakedness (the lower the kurtosis, the more flattened the histogram); "skewness" is related to histogram symmetry (positive skewness indicates a right-tailed histogram); and "entropy" is a metric positively associated with image heterogeneity.

\section{Statistical Analysis}

Descriptive statistics were used to summarize patient and tumor characteristics. Cox proportional hazards regression was used for survival analysis. Univariate analysis was used first, and parameters with significant $P$ values in univariable analysis were used as independent variables in the multivariate analysis. Kaplan-Meier curves were used to graphically display survival in different subgroups. The primary end point was progression-free survival (PFS; recurrence or death was considered to be a dependent event); the secondary end point was overall survival (OS; death from any cause was considered to be a dependent event). Finally,

\section{Image Characteristics and Survival Analysis}

Seventeen patients died during the follow-up, while 10 were alive with progression at 2-year follow-up.

As shown in an earlier study, the mean ADC was significantly lower in HPV-positive patients compared with HPV-negative ones $(P$ value $<.001) .{ }^{12} \mathrm{HPV}$ status and ADC were the only parameters significantly correlated with PFS in univariate analysis, and only HPV status remained significantly associated with PFS in the multivariate analysis, even if it lost significance after correction for the false discovery rate. Only HPV status was associated with OS (Table 2); it also lost significance after correction. Kaplan-Meier curves were plotted for PFS (Fig 2) and OS (Fig 3) using HPV status as a dichotomic variable and for PFS with a mean ADC split by its median value (Fig 4).

At multivariate analysis, only HPV status maintained a statistically significant association with PFS, even if it lost its significance after the Benjamini-Hochberg correction (Table 2). Conversely, the mean ADC was not significantly associated with survival, meaning that the association between ADC and HPV status masked the significance of HPV as a unique independent factor related to survival. 


\begin{tabular}{lcccc}
\hline & HR & $\mathbf{9 5 \%}$ CI & $P$ Value & Corrected $\boldsymbol{P}$ Value \\
\hline PFS, univariate analysis & & & & \\
HPV & 0.27 & $0.11-0.67$ & .01 & .03 \\
Mean ADC (961.37 \pm 184$)$ & 1.00 & $1.00-1.00$ & .01 & .03 \\
SD ADC (186.64 \pm 54.32$)$ & 1.00 & $0.9966-1.0115$ & .29 & .44 \\
Kurtosis ADC (2.08 \pm 2.17$)$ & 1.08 & $0.87-1.34$ & .50 & .50 \\
Skewness ADC (0.86 \pm 0.76$)$ & 0.81 & $0.45-1.45$ & .48 & .50 \\
Entropy ADC (184 \pm 0.5$)$ & 1.90 & $0.78-4.67$ & .16 & .32 \\
OS, univariate analysis & & & & .10 \\
HPV & 0.21 & $0.06-0.76$ & .02 & .34 \\
Mean ADC & 1.00 & $0.999-1.004$ & .2 & .34 \\
SD ADC & 0.1 & $0.985-1.005$ & .34 & .34 \\
Kurtosis ADC & 1.15 & $0.89-1.15$ & .3 & .34 \\
Skewness ADC & 0.69 & $0.35-1.35$ & .28 & .23 \\
Entropy ADC & 2.75 & $0.9-8.43$ & .08 & \\
\hline
\end{tabular}

Note:-HR indicates hazard ratio.

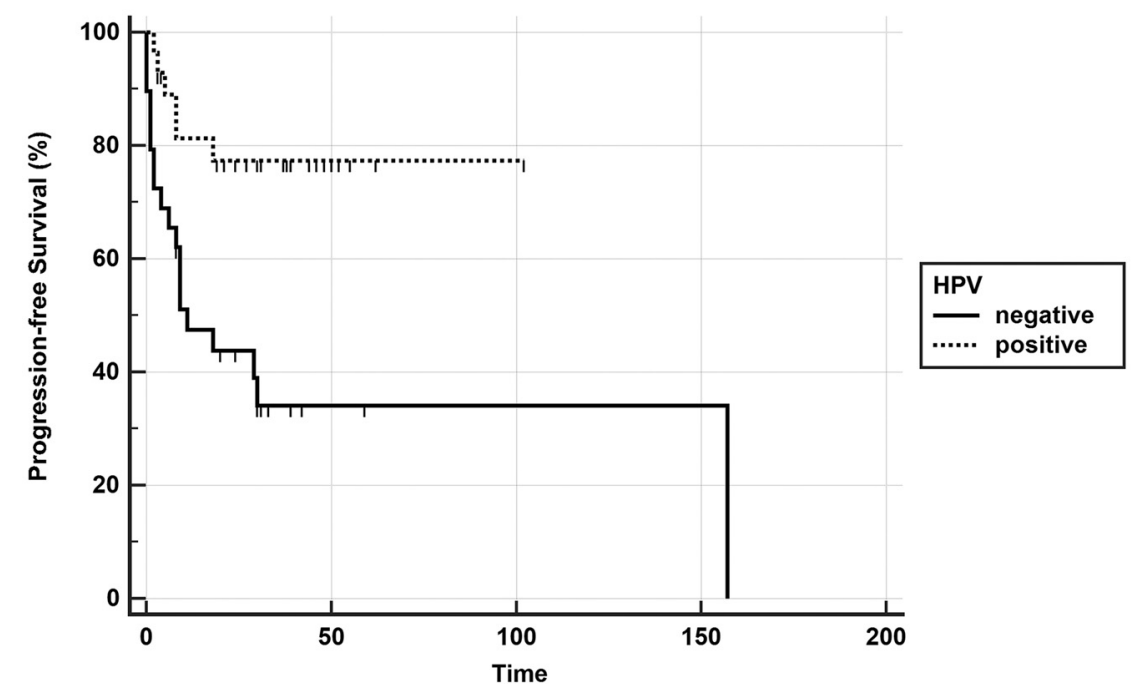

FIG 2. Kaplan-Meier curve showing better progression-free survival in the HPV-positive group ( $P$ value, log-rank test $<$.0001).

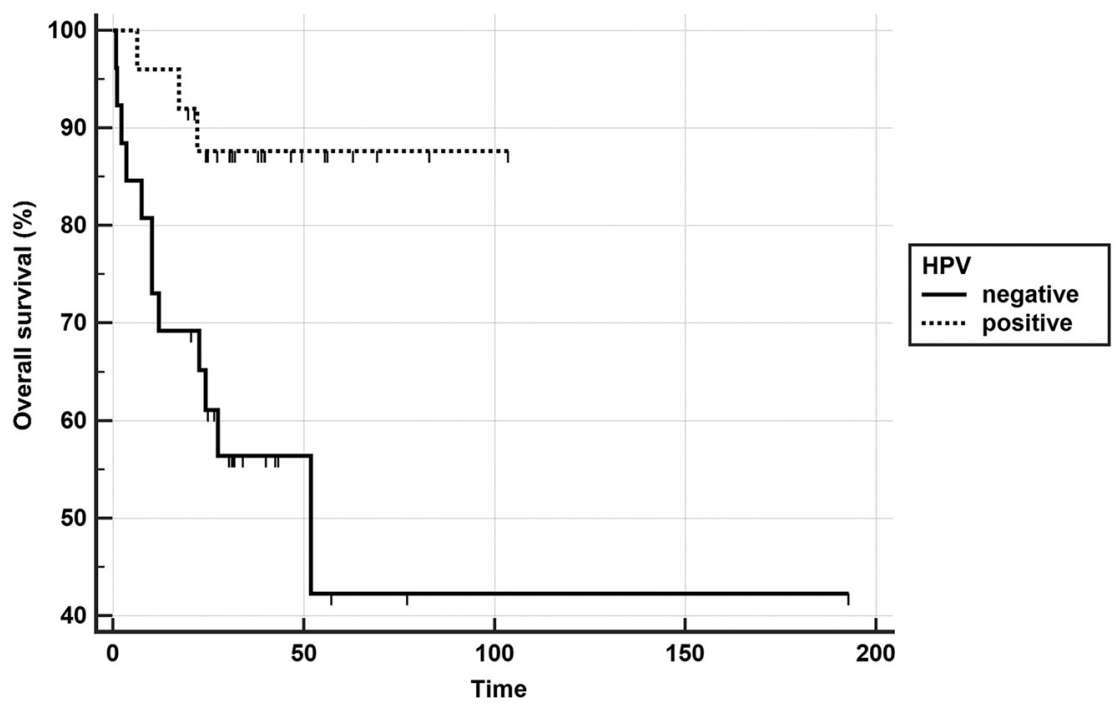

FIG 3. Kaplan-Meier curve showing better overall survival in the HPV-positive group ( $P$ value, log-rank test $<.0001$ ). 


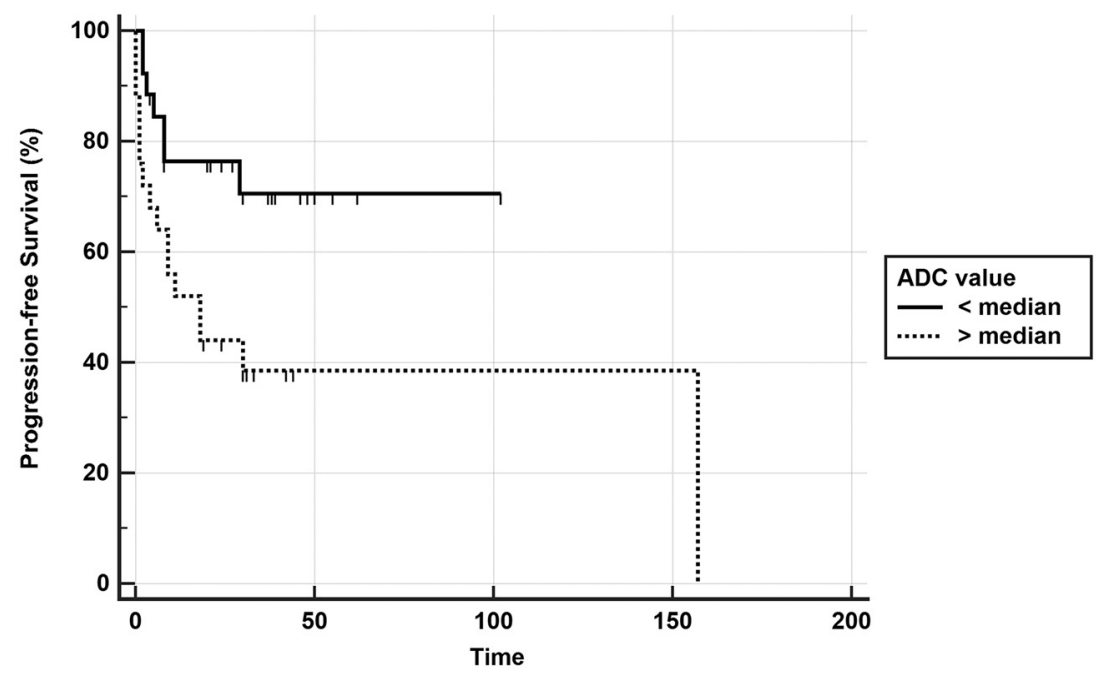

FIG 4. Kaplan-Meier curve showing better progression-free survival in the group with ADC lower than the median value ( $P$ value, log-rank test $<.0001)$.

Table 3: Survival analysis for PFS and OS, dividing the group on the basis of HPV status ADC HPV-Positive $P$ Value HPV-Negative $P$ Value

\begin{tabular}{lll}
\hline DFS & .27 & \\
Mean $(961.37 \pm 184)$ & .79 & .40 \\
SD $(186.64 \pm 54.32)$ & .17 & .79 \\
Kurtosis $(2.08 \pm 2.17)$ & .53 & .98 \\
Skewness $(0.86 \pm 0.76)$ & .63 & .79 \\
Entropy $(184 \pm 0.5)$ & & .24 \\
OS & .14 & .37 \\
Mean & .24 & .13 \\
SD & .57 & .14 \\
Kurtosis & .06 & .65 \\
Skewness & .19 & $.01^{\mathrm{a}}$ \\
Entropy & & \\
\hline
\end{tabular}

Note:-DFS indicates disease-free survival.

${ }^{\mathrm{a}} \mathrm{OR}=4.8461 ; 95 \% \mathrm{Cl}, 1.3507-17.3868$. expected, a positive correlation was found between outcome and HPV status. Moreover, in line with the cited literature, a negative correlation of ADC with disease-free survival was found in the univariate analysis. Other histogram parameters calculated on ADC maps did not correlate with survival in the global group of patients; however, when a multivariate analysis was performed considering mean ADC and HPV status as covariates, only the latter variable maintained its association with survival (however, it was lost after correction to minimize the false discovery rate). The explanation for this result seems to be quite straightforward: The variable with true correlation with outcome is HPV status, while the influence of ADC is likely due to its correlation with HPV status.

When we separated HPV-positive and HPV-negative patients into distinct subgroups, the mean ADC did not correlate with survival. In the univariate analysis, only ADC entropy correlated $(P=.01)$ with survival in the HPV-negative group. Even if, at the moment, the small sample size might cast some doubt on the credibility of this result, it could be hypothesis-generating research for a larger sample size of HPV-negative tumors.

Table 4: Studies investigating the relationship between pretreatment ADC and outcome after chemoradiation therapy in head and neck cancer

\begin{tabular}{|c|c|c|c|c|c|}
\hline Reference & $\begin{array}{c}\text { Year of } \\
\text { Publication }\end{array}$ & $\begin{array}{l}\text { No. of } \\
\text { Patients }\end{array}$ & $\begin{array}{l}\text { No. of Patients with } \\
\text { OPSCC }\end{array}$ & $\begin{array}{l}\text { HPV Status } \\
\text { Available }\end{array}$ & $\begin{array}{c}\text { High ADC Associated } \\
\text { with }\end{array}$ \\
\hline Kim et $\mathrm{al}^{15}$ & 2009 & 33 & 22 & $\mathrm{~N}$ & Poor outcome \\
\hline Hatakenaka et $\mathrm{al}^{16}$ & 2011 & 57 & 22 & $\mathrm{~N}$ & Poor outcome \\
\hline Ohnishi et $\mathrm{al}^{17}$ & 2011 & 64 & 30 & $\mathrm{~N}$ & Poor outcome \\
\hline Srinivasan et $\mathrm{al}^{18}$ & 2012 & 20 & 11 & $\mathrm{~N}$ & Poor outcome \\
\hline Nakajo et $a^{19}$ & 2012 & 26 & 8 & $\mathrm{~N}$ & Better outcome \\
\hline King et $\mathrm{al}^{20}$ & 2013 & 37 & 14 & $\mathrm{~N}$ & Poor outcome \\
\hline Chawla et $a^{21}$ & 2013 & 32 & 9 & $\mathrm{~N}$ & Poor outcome \\
\hline Lambrecht et $\mathrm{al}^{22}$ & 2014 & 161 & 85 & $\mathrm{~N}$ & Poor outcome \\
\hline $\mathrm{Ng}$ et $\mathrm{al}^{23}$ & 2016 & 69 & 37 & $\mathrm{~N}$ & Poor outcome \\
\hline Noij et $\mathrm{al}^{24}$ & 2015 & 78 & 40 & $\mathrm{~N}$ & Poor outcome \\
\hline Preda et $\mathrm{al}^{25}$ & 2016 & 57 & 11 & $\mathrm{~N}^{\mathrm{a}}$ & Poor outcome \\
\hline Marzi et $\mathrm{al}^{26}$ & 2017 & 34 & 14 & $N / Y^{b}$ & Poor outcome \\
\hline Lombardi et $\mathrm{al}^{27}$ & 2017 & 47 & 19 & $\mathrm{~N}$ & Poor outcome \\
\hline Martens et $\mathrm{al}^{28}$ & 2019 & 134 & 96 & Y & Poor outcome $e^{c}$ \\
\hline
\end{tabular}

Note:-N indicates no; $Y$, yes.

${ }^{a}$ Minimum ADC value within the ROI.

${ }^{b}$ Only for the small cohort of patients with OPSCC.

${ }^{c}$ Maximum ADC value within the ROI. 
Recently, Martens et $\mathrm{al}^{28}$ published a well-organized article using ADC histogram analysis to predict HPV status and patient outcome. In agreement with our results, they found a significantly lower ADC value in HPV-positive lesions ( $P$ value $<$ .001). On the other hand, they found that the maximum value of ADC within lesions was negatively correlated with outcome, independent of HPV status and TNM $(P=.024)$. They hypothesized that areas of high ADC could reflect intratumoral necrosis, which is associated with a poorer prognosis. Unlike their study, we have not calculated the maximum ADC; furthermore, they did not find any correlation between outcome and mean ADC, which was a strong predictor of disease-free survival in our study.

Even though they cannot be easily compared with other studies, our results may provide new insights and permit a critical evaluation of previous results. Furthermore, our results demonstrate that OPSCC should be considered a separate entity in studies involving diffusion-weighted imaging as a possible biomarker for outcome prediction, and knowledge of HPV status is mandatory to provide reliability.

Our study has some limitations. First, its retrospective design did not allow some potentially relevant clinical or laboratory variables to be collected. Second, the sample size, even though comparable with that of other literature studies, was low. Third, a study of power to detect the correct number of patients to be enrolled has not been performed. Fourth, ADC was measured on a single central section, which may not be fully representative of the whole tumor.

\section{CONCLUSIONS}

This study demonstrates that ADC is related to disease-free survival after chemoradiation in patients affected by advanced OPSCC. However, this correlation seems to result from the strong association between ADC and HPV status, the latter representing a well-known predictor of survival. Because of these findings, the role of DWI in stratification of OPSCC and, more generally, of head and neck cancer should be revised and likely underestimated. These findings could permit a critical revision of the previous literature and the design of future studies aimed at testing diffusion-weighted imaging as a biomarker of survival in these patients.

Disclosures: Marco Ravanelli-RELATED: Payment for Writing or Reviewing the Manuscript: Linguistic Editing; UNRELATED: Payment for Manuscript Preparation: Linguistic Editing. Paolo Bossi-RELATED: Consulting Fee or Honorarium; UNRELATED: Consultancy: Galera Therapeutics, Comments: less than $\epsilon 1500$. Davide Farina_UNRELATED: Payment for Lectures Including Service on Speakers Bureaus: Marco Ravanelli 2019, Comments: around $\epsilon 1000$; Payment for Development of Educational Presentations: Bracco 2020, Comments: around $\epsilon 1000$.

\section{REFERENCES}

1. Chaturvedi AK, Engels EA, Pfeiffer RM, et al. Human papillomavirus and rising oropharyngeal cancer incidence in the United States. J Clin Oncol 2011;29:4294-4301 CrossRef Medline

2. Huang SH, O'Sullivan B. Overview of the 8th Edition TNM Classification for Head and Neck Cancer. Curr Treat Options Oncol 2017;18:40 CrossRef Medline

3. Würdemann N, Wagner S, Sharma SJ, et al. Prognostic impact of AJCC/UICC 8th Edition new staging rules in oropharyngeal squamous cell carcinoma. Front Oncol 2017;7:129 CrossRef Medline
4. Zanoni DK, Patel SG, Shah JP. Changes in the 8th Edition of the American Joint Committee on Cancer (AJCC) Staging of Head and Neck Cancer: rationale and implications. Curr Oncol Rep 2019;21:52 CrossRef Medline

5. Windon MJ, D'Souza G, Fakhry C. Treatment preferences in human papillomavirus-associated oropharyngeal cancer. Future Oncol 2018;14:2521-30 CrossRef Medline

6. Larsen CG, Jensen DH, Carlander AL, et al. Novel nomograms for survival and progression in HPV + and HPV - oropharyngeal cancer: a population-based study of 1,542 consecutive patients. Oncotarget 2016;7:71761-72 CrossRef Medline

7. Fakhry C, Zhang Q, Nguyen-Tân PF, et al. Development and validation of nomograms predictive of overall and progression-free survival in patients with oropharyngeal cancer. J Clin Oncol 2017;35:4057-65 CrossRef Medline

8. Bossi P, Miceli R, Granata $\mathrm{R}$, et al. In reply to Fakhry et al. Int J Radiat Oncol Biol Phys 2018;102:670-71 CrossRef Medline

9. Leijenaar RT, Carvalho S, Hoebers FJ, et al. External validation of a prognostic CT-based radiomic signature in oropharyngeal squamous cell carcinoma. Acta Oncol 2015;54:1423-29 CrossRef Medline

10. M.D. Anderson Cancer Center Head and Neck Quantitative Imaging Working Group. Investigation of radiomic signatures for local recurrence using primary tumor texture analysis in oropharyngeal head and neck cancer patients. Sci Rep 2018;8:1524 CrossRef Medline

11. King AD, Thoeny HC. Functional MRI for the prediction of treatment response in head and neck squamous cell carcinoma: potential and limitations. Cancer Imaging 2016;16:23 CrossRef Medline

12. Ravanelli M, Grammatica A, Tononcelli E, et al. Correlation between human papillomavirus status and quantitative MR imaging parameters including diffusion-weighted imaging and texture features in oropharyngeal carcinoma. AJNR Am J Neuroradiol 2018;39:1878-83 CrossRef Medline

13. Driessen JP, Caldas-Magalhaes J, Janssen LM, et al. Diffusionweighted MR imaging in laryngeal and hypopharyngeal carcinoma: association between apparent diffusion coefficient and histologic findings. Radiology 2014;272:456-63 CrossRef Medline

14. Swartz JE, Driessen JP, van Kempen PM, et al. Influence of tumor and microenvironment characteristics on diffusion-weighted imaging in oropharyngeal carcinoma: a pilot study. Oral Oncol 2018;77:9-15 CrossRef Medline

15. Kim S, Loevner L, Quon H, et al. Diffusion-weighted magnetic resonance imaging for predicting and detecting early response to chemoradiation therapy of squamous cell carcinomas of the head and neck. Clin Cancer Res 2009;15:986-94 CrossRef Medline

16. Hatakenaka M, Nakamura K, Yabuuchi H, et al. Pretreatment apparent diffusion coefficient of the primary lesion correlates with local failure in head-and-neck cancer treated with chemoradiotherapy or radiotherapy. Int J Radiat Oncol Biol Phys 2011;81:339-45 CrossRef Medline

17. Ohnishi K, Shioyama Y, Hatakenaka M, et al. Prediction of local failures with a combination of pretreatment tumor volume and apparent diffusion coefficient in patients treated with definitive radiotherapy for hypopharyngeal or oropharyngeal squamous cell carcinoma. J Radiat Res 2011;52:522-30 CrossRef Medline

18. Srinivasan A, Chenevert TL, Dwamena BA, et al. Utility of pretreatment mean apparent diffusion coefficient and apparent diffusion coefficient histograms in prediction of outcome to chemoradiation in head and neck squamous cell carcinoma. J Comput Assist Tomogr 2012;36:131-37 CrossRef Medline

19. Nakajo M, Nakajo M, Kajiya Y, et al. FDG PET/CT and diffusionweighted imaging of head and neck squamous cell carcinoma: comparison of prognostic significance between primary tumor standardized uptake value and apparent diffusion coefficient. Clin Nucl Med 2012;37:475-80 CrossRef Medline

20. King AD, Chow KK, Yu KH, et al. Head and neck squamous cell carcinoma: diagnostic performance of diffusion-weighted MR imaging for the prediction of treatment response. Radiology 2013;266:531-38 CrossRef Medline 
21. Chawla S, Kim S, Dougherty L, et al. Pretreatment diffusionweighted and dynamic contrast-enhanced MRI for prediction of local treatment response in squamous cell carcinomas of the head and neck. AJR Am J Roentgenol 2013;200:35-43 CrossRef Medline

22. Lambrecht M, Van Calster B, Vandecaveye V, et al. Integrating pretreatment diffusion weighted MRI into a multivariable prognostic model for head and neck squamous cell carcinoma. Radiother Oncol 2014;110:429-34 CrossRef Medline

23. Ng SH, Liao CT, Lin CY, et al. Dynamic contrast-enhanced MRI, diffusion-weighted MRI and 18F-FDG PET/CT for the prediction of survival in oropharyngeal or hypopharyngeal squamous cell carcinoma treated with chemoradiation. Eur Radiol 2016;26:4162-72 CrossRef Medline

24. Noij DP, Pouwels PJ, Ljumanovic R, et al. Predictive value of diffusion-weighted imaging without and with including contrastenhanced magnetic resonance imaging in image analysis of head and neck squamous cell carcinoma. Eur J Radiol 2015;84:108-16 CrossRef Medline

25. Preda L, Conte G, Bonello L, et al. Combining standardized uptake value of FDG-PET and apparent diffusion coefficient of DW-MRI improves risk stratification in head and neck squamous cell carcinoma. Eur Radiol 2016;26:4432-41 CrossRef Medline

26. Marzi S, Piludu F, Sanguineti G, et al. The prediction of the treatment response of cervical nodes using intravoxel incoherent motion diffusion-weighted imaging. Eur J Radiol 2017;92:93-102 CrossRef Medline
27. Lombardi M, Cascone T, Guenzi E, et al. Predictive value of pretreatment apparent diffusion coefficient (ADC) in radio-chemiotherapy treated head and neck squamous cell carcinoma. RadiolMed 2017;122:345-52 CrossRef Medline

28. Martens RM, Noij DP, Koopman T, et al. Predictive value of quantitative diffusion-weighted imaging and 18-F-FDG-PET in head and neck squamous cell carcinoma treated by (chemo)radiotherapy. Eur J Radiol 2019;113:39-50 CrossRef Medline

29. Chan MW, Higgins K, Enepekides D, et al. Radiologic differences between human papillomavirus-related and human papillomavirusunrelated oropharyngeal carcinoma on diffusion-weighted imaging. ORL J Otorhinolaryngol Relat Spec 2016;78:344-52 CrossRef Medline

30. Nakahira M, Saito N, Yamaguchi H, et al. Use of quantitative diffusion-weighted magnetic resonance imaging to predict human papilloma virus status in patients with oropharyngeal squamous cell carcinoma. Eur Arch Otorhinolaryngol 2014;271:1219-25 CrossRef Medline

31. Schouten CS, de Graaf P, Bloemena E, et al. Quantitative diffusionweighted MRI parameters and human papillomavirus status in oropharyngeal squamous cell carcinoma. AJNR Am J Neuroradiol 2015;36:763-67 CrossRef Medline

32. de Perrot T, Lenoir V, Domingo Ayllón M, et al. Apparent diffusion coefficient histograms of Human Papillomavirus-positive and human papillomavirus-negative head and neck squamous cell carcinoma: assessment of tumor heterogeneity and comparison with histopathology. AJNR Am J Neuroradiol 2017;38:2153-60 CrossRef Medline 